This is the final peer-reviewed accepted manuscript of:

L. Bottaccioli et al., "A Flexible Distributed Infrastructure for Real-Time Cosimulations in Smart Grids," in IEEE Transactions on Industrial Informatics, vol. 13, no. 6, pp. 3265-3274, Dec. 2017

The final published version is available online at:

https://doi.org/10.1109/TII.2017.2702206

Rights / License:

The terms and conditions for the reuse of this version of the manuscript are specified in the publishing policy. For all terms of use and more information see the publisher's website.

This item was downloaded from IRIS Università di Bologna (https://cris.unibo.it/)

When citing, please refer to the published version. 


\title{
A Flexible Distributed Infrastructure for Real-Time Co-Simulations in Smart Grids
}

\author{
Lorenzo Bottaccioli, Abouzar Estebsari, Enrico Pons, Ettore Bompard, Enrico Macii, Edoardo Patti \\ and Andrea Acquaviva.
}

\begin{abstract}
Due to the increasing penetration of distributed generation, storage, electric vehicles and new ICT technologies, distribution networks are evolving towards the Smart Grid paradigm. For this reason, new control strategies, algorithms and technologies need to be tested and validated before their actual field implementation. In this paper we present a novel modular distributed infrastructure, based on real-time simulation, for multi-purpose Smart Grid studies. The different components of the infrastructure are described and the system is applied to a case study based on a real urban district located in northern Italy. The presented infrastructure is shown to be flexible and useful for different and multi-disciplinary Smart Grid studies.
\end{abstract}

Index Terms-Co-simulation, distributed infrastructure, photovoltaic, Smart Grid, real-time simulation, renewable energy.

\section{INTRODUCTION}

$\mathbf{M}$ ANY countries worldwide are providing incentives for the connection to electricity networks of lowcarbon and sustainable generation technologies, in particular photovoltaic (PV) systems, especially following the Kyoto protocol [1]. These renewable and sustainable generation technologies are often connected to electricity distribution networks in the form of distributed generation (DG), at the low voltage (LV) or medium voltage (MV) level.

The introduction of DG, which can be based on renewable or on conventional sources, substantially modifies the operation of electricity networks: the direction of power flows in the MV lines and even in high voltage/medium voltage (HV/MV) transformers can be reversed, voltage profiles are modified, fault management is affected [2], [3], etc.

In addition to this, electric vehicles are also forecasted to become widespread in the near future, modifying the consumption patterns in the networks and providing possible services to Distribution System Operators (DSOs) for network management [4]. Also stationary storage is becoming economically convenient and is being tested in some pilot networks by DSOs.

Moreover, DSOs are also enhancing the ICT (Information Communication Technologies) layer used to monitor and control distribution networks.

L. Bottaccioli E. Macii, E. Patti and A. Acquaviva are with the Department of Control and Computer Engineering, Politecnico di Torino, Italy.

A. Estebsari, E. Pons and E. Bompard are with the Department of Energy, Politecnico di Torino, Italy.

Emails: \{lorenzo.bottaccioli, abouzar.estebsari, enrico.pons, ettore.bompart, enrico.macii, edoardo.patti, andrea.acquaviva\}@polito.it

This work was partially supported by the EU project FLEXMETER, and by the Italian project "Edifici a Zero Consumo Energetico in Distretti Urbani Intelligenti”.

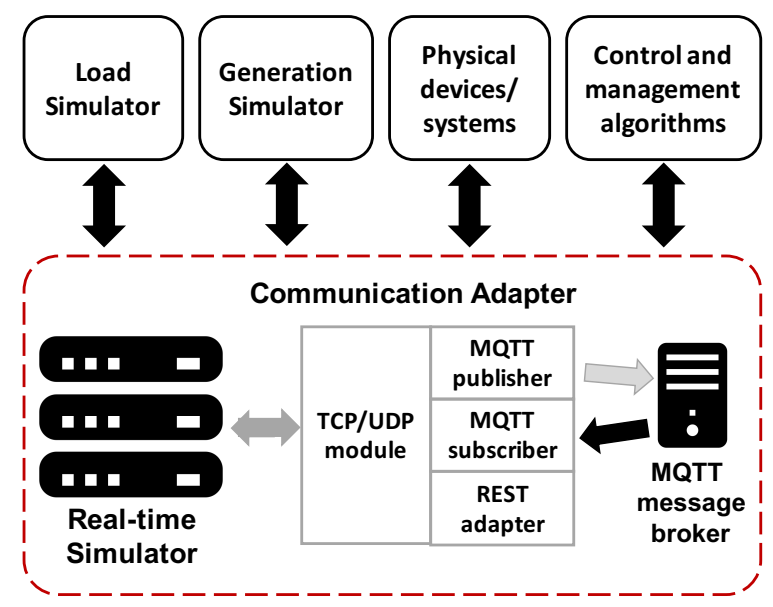

Fig. 1. Scheme of the proposed distributed infrastructure for real-time cosimulations in smart grids

For all these reasons, distribution networks need to become Smart and new control strategies, algorithms and technologies need to be tested and validated before their implementation and installation in real systems.

In this paper, we present a novel, multi-purpose, distributed infrastructure for real-time simulations in Smart Grid scenarios. This tool is modular and is constituted by six main blocks, as depicted in Figure 1, but could be extended in the future. The core of the infrastructure is a real-time simulator, which can simulate the network behaviour. Through a Communication Adapter, the real-time simulator is interconnected to external modules which provide different functionalities, e.g. the simulation of generation, loads, algorithms and the possibility to interconnect physical devices or systems. All these modules will be deeply described in Section III.

The main advantage of the implemented infrastructure is its capability to simulate new systems, which are different from a previous test in terms of input data or type of model, with a minimum effort. Any changes in the input data of the modules (i.e. new consumption profiles; new grid; or new generation scenario) as well as changes in the type or conceptual model of a module (e.g. new prosumer behaviour model; other types of generators, such as PV, wind, fuel cells; and new control and management algorithms) require a minimum effort to be implemented without creating the entire simulation model from scratch. In addition, the proposed infrastructure enables to study the interoperability among different use-cases in a plug-play fashion. Finally, the proposed solution can integrate Smart Metering Architecture to exploit (near-) real-time data 
collected from the field to co-simulate different smart energy strategies with real information. These are the strengths of the proposed solution: the platform is flexible and open to include, replace, or enhance the modules for any new use cases/scenarios.

The rest of this paper is organized as follows. Section II reviews most relevant literature solutions. Section III introduces the methodology and the main actors (see Figure 1) we identified for co-simulations in Smart Grids. Following this methodology, we developed the proposed distributed infrastructure that is presented in Section IV. Section V shows possible applications and demonstrates the usefulness of the proposed tool thanks to an application example. The case study and the experimental results are focused on the distributed control of storages connected to the distribution grid. Finally, Section VI discusses concluding remarks.

\section{RELATED WORK}

Smart Grids are complex systems where different entities cooperate by exchanging heterogeneous information. In this view, Bompard et al. [5] theoretically conceive a multilayer system with: i) a Physical layer for including hardware components to monitor and manage the grid; ii) a Cyber layer for defining information flows and managing operations and market-related data-set; iii) a Social layer for including unpredictable performances due to actors that play in power system scenario (i.e. users, prosumers and system operators); and iv) an Environment layer for integrating natural phenomena that affects the previous layers.

In [6], authors present INSPIRE, a co-simulation framework for evaluating real-time power systems tougher with ICT. It is based on both the IEC 61850 standard and the IEC $61968 / 61970$ common information model. Thus, INSPIRE simulates the interactions between the Physical layer and Cyber layer.

Yang et al. [7] propose a co-simulation environment for HIL/SIL (Hardware-in-the-loop/Software-in-the-loop) validations of distributed controls in Smart Grid. They designed the controls by exploiting a model-driven approach using the IEC 61499 standard. Controllers are developed in MATLAB/Simulink and communicate with power plants models through UDP (User Datagram Protocol) and TCP (Transmission Control Protocol) sockets to build closed-loop models. Authors highlight that a co-simulation approach is extremely useful in the context of distributed automation.

In [8], authors present a specific HIL/SIL co-simulation platform to verify the performance of a volt-VAR optimization engine for smart distribution networks. In its core, it integrates a real-time simulator for distribution networks with physical devices for Measurement \& Control. For message exchanges in the platform, they implemented both IEC 61850 MMS and GOOSE protocols.

Another example of multi-layer co-simulation platform for complex-system analysis is presented in our previous works [9], [10]. This solution is devoted only to evaluate the impacts of photovoltaic (PV) distributed generation systems, especially in densely populated urban areas. It implements Physical, Cyber and Environmental layers.
MOSAIK [11]-[13] is a flexible architecture consisting of four layers for managing control strategies, scenario specifications and simulation models exploiting semantic knowledge. In particular, to perform power-flow analysis in a smart grid scenario, [13] integrates a co-simulation between the software PowerFactory with their model (in Matlab) for both PV and Load generations. GRIDspice [14] is a distributed simulation platform that integrates existing simulation software for power generation and distribution (i.e. MATPOWER and Gridlab-D). Furthermore, GRIDspice exploits a cloud-based architecture that allows the parallelization of large simulation jobs. SGsim [15] integrates two tools for simulating both power and communication flows to co-simulate smart grid applications, such as Conservation Voltage Reduction. The three co-simualation tools MOSAIK, GRIDspice and SGsim offer a flexible and scalable simulation framework but limited to SIL, without the possibility of HIL. Furthermore, they do not provide real-time features for short-transient phenomena.

In [16] the authors presents a co-simulation testbed for microgrids. They integrate a RTS with a communication emulator in order to test control algorithms for micro-grid.

In the framework of smart grids real-time simulation, ENEL, the biggest Italian utility, is running test and validation projects in some of its smart grids laboratories. POI P3 Smart Grid project is an example of these activities [17], with the purpose of testing new techniques for voltage regulation through SCADA and DMS. All devices and systems in this project communicate only via IEC 61850 standard. Lack of forecasted power-time profiles of the loads and generators was the other limitation of its test set up implementation.

Finally in [18], authors presents a flexible co-simulation environment that exploits an IoT approach. Indeed, information from IoT devices (e.g. smart meters) has feed into a realtime simulator through the publish/subscribe communication paradigm [19].

The presented literature solutions [7]-[10] have been designed and implemented to address a single use-case or scenario with specific requirements, while such co-simulation platforms should be designed to be flexible in simulating different use cases and scenarios in power grids. In addition, they should integrate Internet-of-Things (IoT) communication paradigms and protocols to provide flexibility and scalability in performing HIL and SIL co-simulations. Thus, they will be ready to enable the communication among next generation devices (e.g. Smart Meters), that will be IoT devices. On this premises, we propose a distributed infrastructure for realtime co-simulations in Smart Grids. It exploits communication paradigms peculiar of IoT platforms, to implement a flexible framework where different power grid scenarios and simulations can be executed. It includes Physical, Cyber and Environmental layers depicted in [5] providing features to perform both HIL and SIL co-simulations. In addition, it can also integrate Smart Metering Infrastructure (e.g. [20], [21]) to retrieve measurements from real devices deployed along the distribution network.

To highlight our contribution, Table I reports a comparison of our solution with the reviewed smart-grid co-simulation environments. It highlights: i) what are the simulation layers 


\begin{tabular}{|c|c|c|c|c|c|c|}
\hline & $\begin{array}{l}\text { Integrated } \\
\text { layers }\end{array}$ & Co-Simulation & RTS & $\begin{array}{l}\text { Use-case } \\
\text { Scenario }\end{array}$ & HIL/SIL & IoT \\
\hline \multirow[t]{2}{*}{ INSPIRE [6] } & $\begin{array}{l}\text { Physical } \\
\text { Cyber }\end{array}$ & $\begin{array}{l}\text { Real-time co-simulation } \\
\text { of Cyber-physical } \\
\text { energy-systems }\end{array}$ & $\mathrm{x}$ & Multiple & $\mathrm{x}$ & $\mathrm{x}$ \\
\hline & $\begin{array}{l}\text { Physical } \\
\text { Cyber }\end{array}$ & $\begin{array}{l}\text { Real-time co-simulation } \\
\text { between two simulation } \\
\text { environments (MATLAB } \\
\text { and Function Block) }\end{array}$ & $\mathrm{x}$ & Single & $\begin{array}{l}\text { HIL } \\
\text { SIL }\end{array}$ & $\mathrm{x}$ \\
\hline Manbachi et al. [8] & $\begin{array}{l}\text { Physical } \\
\text { Cyber }\end{array}$ & $\begin{array}{l}\text { Real-time co-simulation } \\
\text { of grid status and } \\
\text { volt variation controllers }\end{array}$ & $\checkmark$ & Single & $\begin{array}{l}\text { HIL } \\
\text { SIL }\end{array}$ & $\mathrm{x}$ \\
\hline Bottaccioli et al. [9], [10] & $\begin{array}{l}\text { Physical } \\
\text { Cyber } \\
\text { Environmental }\end{array}$ & $\begin{array}{l}\text { Real-time co-simulation } \\
\text { of PV energy production } \\
\text { and grid status }\end{array}$ & $\checkmark$ & Single & $\begin{array}{l}\text { HIL } \\
\text { SIL }\end{array}$ & $\mathrm{x}$ \\
\hline MOSAIK [11]-[13] & $\begin{array}{l}\text { Physical } \\
\text { Cyber }\end{array}$ & $\begin{array}{l}\text { Co-simulation of power } \\
\text { flow and load generation }\end{array}$ & $\mathrm{x}$ & Multiple & SIL & $\mathrm{x}$ \\
\hline GRIDspice [14] & $\begin{array}{l}\text { Physical } \\
\text { Cyber }\end{array}$ & $\begin{array}{l}\text { Co-simulation of power- } \\
\text { and communication-flows } \\
\text { in smart-grids }\end{array}$ & $\mathrm{x}$ & Multiple & SIL & $\mathrm{x}$ \\
\hline SGsim [15] & $\begin{array}{l}\text { Physical } \\
\text { Cyber }\end{array}$ & $\begin{array}{l}\text { Co-simulation of power- } \\
\text { and communication-flows } \\
\text { for smart-grids application } \\
\text { such as CVR }\end{array}$ & $\mathrm{x}$ & Multiple & SIL & $\mathrm{x}$ \\
\hline Hahn et al. [16] & $\begin{array}{l}\text { Physical } \\
\text { Cyber }\end{array}$ & $\begin{array}{l}\text { Real-time co-simulation } \\
\text { of power- and } \\
\text { communication-flows } \\
\text { to test control algorithms } \\
\text { in micro-grids }\end{array}$ & $\checkmark$ & Multiple & $\begin{array}{l}\text { HIL } \\
\text { SIL }\end{array}$ & $\mathrm{x}$ \\
\hline ENEL [17] & $\begin{array}{l}\text { Physical } \\
\text { Cyber }\end{array}$ & $\begin{array}{l}\text { Real-time simulation } \\
\text { of protection and } \\
\text { automation strategies }\end{array}$ & $\checkmark$ & Multiple & $\begin{array}{l}\text { HIL } \\
\text { SIL }\end{array}$ & $\mathrm{x}$ \\
\hline Our solution & $\begin{array}{l}\text { Physical } \\
\text { Cyber } \\
\text { Environmental }\end{array}$ & $\begin{array}{l}\text { Real-time simulation } \\
\text { of smart grid control } \\
\text { algorithms }\end{array}$ & $\checkmark$ & Multiple & $\begin{array}{l}\mathrm{HIL} \\
\mathrm{SIL}\end{array}$ & $\checkmark$ \\
\hline
\end{tabular}

TABLE I

COMPARISON AMONG OUR CO-SIMULATION INFRASTRUCTURE AND LITERATURE SOLUTIONS.

among Physical, Cyber and Environmental that each solution integrates in the simulation environment; ii) what kind of co-simulation is performed; iii) if a Real-time simulator is used; iv) if the solution simulates single or multiple usecases/scenarios; v) if HIL and/or SIL simulations can be performed; and vi) if the simulation environment interacts with IoT devices.

The strength of this infrastructure comes from its capability to host new different use cases, input data, or models by means of its modules in a plug-and-play fashion, with a minimum effort to change the overall setup. As an example, several distributed generations (DGs) can be integrated as either independent simulators or standalone physical generators thanks to our IoT-based architecture which is a novel contribution to the co-simulations of electric energy systems with respect to conventional setups.

\section{DiSTRIBUTED INFRASTRUCTURE FOR SIMULATING SMART GRID BEHAVIOURS}

In this Section, we describe the components of the proposed distributed infrastructure (see Figure 1). Then, in Section IV, we present as an example our implementation for a decentralized management of battery storage to maximize the selfsufficiency of the grid with PV generation.

As shown in Figure 1, we identify six main distributed components for the proposed infrastructure: i) Real-Time Simulator, ii) Communication Adapter, iii) Load Simulator, iv) Generation Simulator, v) Physical Devices/Systems and vi) Control and management algorithms. The rest of this Section describes each component of the proposed infrastructure in more detail.

\section{A. Real-Time Simulator}

The Real-Time Simulator (RTS) is in charge of reproducing the behaviour of a real electric distribution system, which is modelled as a test bed for testing and validation of new technologies, management algorithms or control strategies, through performing software in-the-loop (SIL) or hardware in-the-loop (HIL).

In SIL, an algorithm or a control strategy is tested on a realtime model of the system. In this way, validation is carried out safely, and under a variety of different scenarios which may not be all feasible or available in real world (e.g. faulty conditions). In HIL similar tests can be performed, connecting physical devices to the simulated environment.

In our proposed architecture, the real-time simulation module is mainly in charge of reproducing the distribution grid behavior from physical infrastructure perspective. In other words, physical components of the grid including generators, transformers, lines, breakers, loads, etc. are modeled in RTS, while the models which generate time-variant inputs for loads, generators, or controllable devices are made and hosted outside. The grid model can be implemented either in RSCAD, in case of using RTDS real-time simulator, or in MATLAB/Simulink, in case of using OPAL-RT real-time simulator with eMEGAsim configuration for EMT analysis.

Real-time simulation is actually simulating a system, which realistically responds to the external stimuli, as the inputs/outputs of the simulation are synchronous with the real world. Section III-B presents the Communication Adapter, which is our software solution to enable a bidirectional communication between the real-time simulator and other services and/or devices.

For instance, the requested measurements from the realtime model can be sent out through the Communication Adapter to be used by the control and management algorithm development nodes (SIL). In addition, the real-time digital simulator can communicate with real physical devices and system components (e.g. a micro-grid controller, protection automation systems, PV panels and wind turbines) through analog and digital I/O signals (HIL).

In the proposed distributed infrastructure, the RTS models the distribution system, receives inputs from external modules for single components modelling and provides the required measurements or signals to external applications. It actually provides simulation as a service.

\section{B. Communication Adapter}

The Communication adapter (see Figure 1) is in charge of enabling data exchange among the Real-time simulator and other infrastructure modules. It implements two communication paradigms: i) publish/subscribe [19] based on MQTT (Message Queuing Telemetry Transport) protocol [22] and ii) request/response based on REST (Representational State Transfer) [23]. In particular, the publish/subscribe communication model allows the development of loosely-coupled event-based systems. As explicit dependencies between dataproducer and data-consumer are removed, each module in the proposed infrastructure can publish data, which can be independently received by a number of subscribers. This also increase the scalability of the whole infrastructure [20]. The request/response approach enables the RTS in retrieving 
information from REST web services, such as our Photovoltaic simulator introduced in Section IV-C.

In its core, the Communication adapter exploits the $T C P / U D P$ module to allow a bidirectional communication with the RTS. It exploits either TCP or UDP protocols to give a full communication compatibility with the real-time simulators in the market (e.g. Opal-RT) that provide software libraries and tools to exploit these two protocols. Thus, depending on the communication requirements needed by the simulation, the Communication adapter can be configured to use either TCP or UDP. Such real-time simulators are in charge of performing hard real-time simulations respecting the predefined timesteps. However, the interaction among different modules of the overall co-simulation for most of the use-cases requires rates of exchanging data in the range of seconds to minutes, which are much higher than the latency of the communication infrastructure. This guarantees that messages are delivered in time. This module implements both server and client functionalities, in that it receives and sends data to the real-time simulation engine. Such data includes either electrical measurement from virtual meters in the grid model (e.g. voltage, current, active and reactive power) or control signals from some control and management algorithms (e.g. voltage or current signals, time-variant load or generation values and switch activation signals). The Communication adapter provides the MQTT publisher module that parses data from RTS into sequence of events before publishing to an MQTT Message broker. The Message broker keeps track of all publications and subscriptions and takes care of sending new data to subscribers. Hence, any client application (e.g. Control and managements algorithms) subscribes to the Message broker for receiving an information flow according to its needs. The Communication adapter works also as subscriber. Therefore, it is able to receive commands from remote applications (e.g. Control and managements algorithms and Load Simulator modules) and pushes them to the real-time simulation engine. Finally, the REST adapter is in charge of retrieving information from REST web service, even third party services, and passes the results to RTS, again through the TCP/UDP module.

The Communication adapter also translates the information from the data-format used by the software components in Figure 1 to the data-format suitable for the RTS and vice-versa. These software components exploit JSON that is an openstandard format that uses human-readable text to transmit data objects consisting of key-value pairs. It is the most common data-format used for data exchange in web environments. Whilst, the data-format for RTS must be a vector of numbers. We organized this vector also as a series of key-value pairs. The odd positions in the vector are the numeric keys while the even positions are the associated numeric values. Thus, the Communication adapter translates each key-value pair in the JSON into the equivalent numeric key-value pair in the vector and vice-versa.

In this platform, real-time simulation is performed by the digital real time simulator in which the virtual model of the grid is simulated. The overall co-simulation is in nearreal-time mode as the data exchange is seldom in real-time due to communication delay of the infrastructure. To ensure

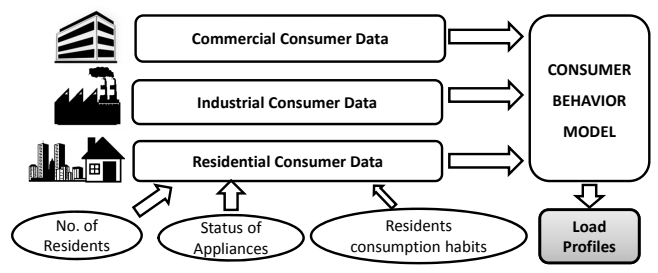

Fig. 2. Overall functionalities and inputs of the Energy load profiles module to generate energy consumption profiles

synchronization, the real-time simulator is running with a shifted time stamp with respect to the real world wall-clock time.

It is worth noting that the Communication adapter allows the integration of the Real-time simulator with other smart metering infrastructure (e.g. [20], [21]), where each smart meter is an Internet-of-Things (IoT) device. Hence, data coming from such devices can be used to simulate and test innovative control strategies with (near-) real-time data from the grid. Vice-versa, each simulated grid component is also seen by other modules as an IoT device able to send information and to receive commands.

\section{Load and Generation Simulators}

For what concerns modelling a distribution system, besides network data such as grid topology, components characteristics and parameters and devices settings, there are some timevariant inputs which should be pushed into the running model. Theses inputs mainly include loads consumption and generators production data. As these inputs can follow different profiles based on simulation scenarios for special studies, in our distributed infrastructure, two stand-alone modules are in charge of providing such inputs, communicating with the grid real-time model.

For this purpose, the platform includes two system components for simulating the load and generation profiles: i) Load Simulator and ii) Generation Simulator.

The load simulator models electrical energy consumption of the grid, such as constant impedance, constant power and constant current, depending on the application and simulation type. Load profiles can be generated with consumer behaviour models (Figure 2) or forecasting tools.

The generation simulator is actually the virtual model of distributed generation units, such as wind farms and PV panels, and reproduces the behaviour of these generators in terms of time-variant active and reactive power. This virtual generation model is affected by environmental conditions. Thus, it implements the Environmental layer depicted by [5].

In the proposed infrastructure, both modules work as publishers to send data via MQTT protocol. These components can be replaced by a smart metering architecture to exploit (near-) real-time data coming from IoT devices deployed in the real electrical distribution network, as mentioned in Section III-B. This replacement can be done without affecting the rest of the proposed infrastructure. 


\section{Physical Devices/Systems}

The proposed real-time co-simulation infrastructure foresees the integration with real devices exploiting two different approaches: i) Hardware in the Loop (HIL) and ii) "Smart Metering Infrastructure in-the-Loop".

HIL refers to the simulations in which a real device (e.g. relay and PV panel) is connected to a virtual environment instead of real system (e.g. simulated grid).

As smart meters can be considered as IoT devices, our proposed architecture interconnects them with the real time simulator (as the grid virtual model) based on an IoT approach, which is different from the conventional off-the-shelf solutions used for HIL. With "Smart Metering Infrastructure in-theLoop", we refer to possible integration of our co-simulation framework with third-party smart metering infrastructure, such as [20], [21]. As mentioned in Section III-B, this integration can be achieved thanks to the Communication adapter. In this view, each internet-connected smart meter sends measurements to its smart metering infrastructure that can forward them to the Real-Time Simulator exploiting either MQTT or REST adapters. Thus, (near-) real-time data from the real distribution network can be used for simulating and testing new algorithms and strategies.

\section{E. Control and Management Algorithms}

Figure 3 shows how the proposed distributed simulation architecture can support decision making for development and improvement of electric distribution systems control and management, especially in emerging smart grids with considerable amount of distributed generation. The virtual model of the grid can be built and simulated in real-time thanks to the available data from real world system. Energy consumption and production profiles can be generated using collected data from the real system through simulations or forecasting tools. A variety of different control or management strategies can be then considered within this framework. The applications could include emerging methodologies in distribution systems automation, distributed and centralized state estimation processes, Volt/VAR regulation, real-time power quality monitoring for distribution, storage management (either vehicleto-grid [24] or stationary), demand response and demand side management, outage management strategies including fault and outage location algorithms, restoration procedures, synchrophasor applications in active distribution networks, etc. Any physical prototype or off-the-shelf device (e.g. protection relay) can be also put in the loop as HIL. Real-time simulation provides real-like time-step snapshots of the system status for ex-post-analysis and application results monitoring. Parameters of the scenarios and control strategy can be tuned and modified iteratively to improve design performance.

\section{INFRASTRUCTURE IMPLEMENTATION}

In this section, we describe the implementation of the proposed distributed infrastructure for real-time simulations in Smart Grid scenarios. A distributed storage managment technique is also implemented as an example use case. The main objective of this use case is to shift existing paradigm

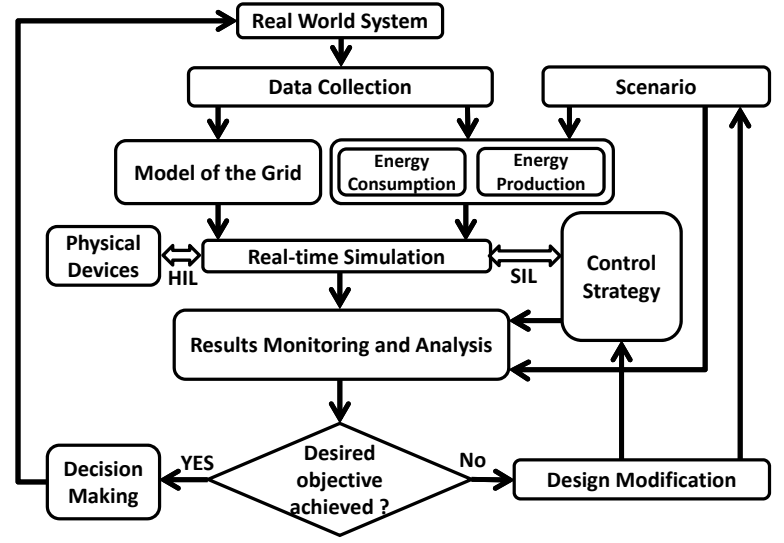

Fig. 3. Flowchart for control strategies development using the proposed infrastructure.

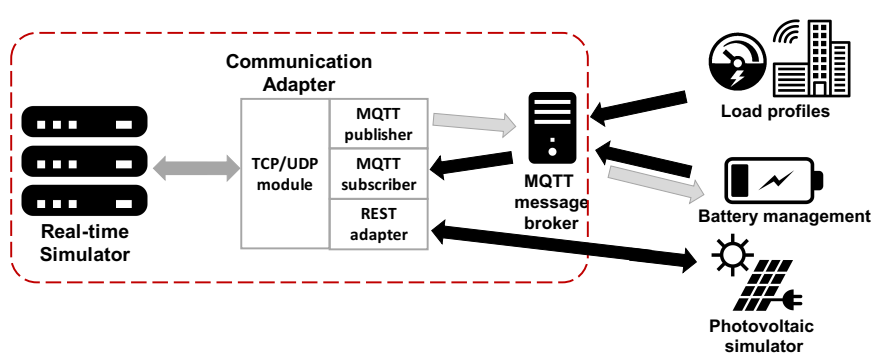

Fig. 4. Scheme of data-flows and command signals exchange in the proposed distributed infrastructure

of energy balance in MV substations towards microgrids. As shown in Figure 4, the co-simulation infrastructure consists of five main modules: i) Real-time simulator, ii) Communication adapter, iii) Load profiles module, iv) Battery management module and v) Photovoltaic simulator. Since it has been designed to be modular, each module can be replaced or updated without affecting the rest of the infrastructure. The current implementation does not include the Physical Devices module, but this can be easily integrated to feed the cosimulation engine. In addition, computers and servers, that host and run the software components of our solution, can optionally exploit the Network Time Protocol (NTP) to be synchronized. Finally, as mentioned in Section III-B and III-D, this infrastructure is ready to integrate other smart metering infrastructures (e.g. [20], [21]), where each smart meter is an IoT device that sends measurements in (near-) real-time. The rest of this section describes each module in more detail.

\section{A. Real-Time Simulator}

In this implementation, we use an Opal RT OP5600 realtime simulator with 12 cores operating at $3.46 \mathrm{GHz}$ and eMEGAsim configuration aimed for electromagnetic transient (EMT) analysis. Electricity grid is modelled in MatLab Simulink using SimPowerSystem (SPS) under SimScape library. An additional library called ARTEMiS provided by Opal-RT is also used to provide fixed-step solver dedicated to complex power systems. The model is compiled using RTLAB software and executed on the simulator. As introduced before, any application algorithm or control/management strat- 


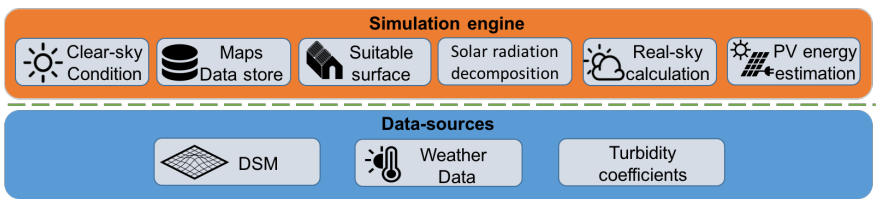

Fig. 5. Scheme of the software architecture for PV systems integration into Smart Grids

egy is kept outside of the real-time model and interfaced through communication channels.

As the external modules are not necessarily synchronized with the real-time model and they run independently of the real-time system, either at their own time-step or launched by external triggers, we use Asynchronous Process Application. In this case, a low speed serial data acquisition (e.g. with some milliseconds or seconds time step) can be performed in a separate process from the real-time model, where a high speed data exchange is required (e.g. $250 \mu s$ time-step).

The asynchronous process requires a $\mathrm{C}$ or $\mathrm{C}++$ source code implemented by the user to handle some sort of communication blocks inside the real-time model. The code consists of some predefined functions which are compiled once during the initialization process. The data signals to be sent or received and the communication protocols (i.e. TCP or UDP) to use for communicating with the Communication Adapter should be also selected inside the real-time model prior to model execution.

\section{B. Load Simulator}

The load profile generator module can be either replaced with a consumer behavior model or a forecasting tool. In our implementation, we used a simple MATLAB script running in a computer as the host of load simulator module in order to test the overall implemented infrastructure. The MATLAB code reads the aggregated active and reactive power of substations from a profile, and periodically (every 15 minutes) publishes a set of data through the MQTT protocol [22]. The profiles are built based on measurements performed by us in real MV/LV substations, and they are scaled considering the transformer capacity and number of buildings.

\section{Photovoltaic system simulator}

The Photovoltaic system simulator (PV-sim) is a software component of the proposed solution to model the behaviour of PV system in urban contexts. It simulates sub-hourly solar irradiance in real-sky conditions to estimate PV systems generation profiles. As shown in Figure 5, it requires heterogeneous inputs from different data-sources, as described in the following. The Digital Surface Model (DSM) is a raster image that represents terrain elevation considering the presence of manufactures. Linke Turbidity coefficients express the attenuation of solar radiation related to air pollution. Weather Data are information on climatic conditions (e.g. solar irradiance, air temperature and wind speed) that are retrieved from (personal) weather stations through third-party services (such as [25]).
The Simulation engine is the core of PV-sim (see Figure 5). It has been designed following the microservice approach [26] composed by different software modules that provide REST web services [23] to run simulations and exchange information. Starting from the monthly Linke Turbidity Coefficients and the DSM for the area of interest, the Clear-Sky condition module provides an accurate simulation of solar radiation in urban contexts. The resulting outputs of this module are set of direct and diffuse solar radiation maps in clear-sky conditions with 15 minutes time interval. Such maps are stored in the Maps Data-store ready to be used by the other components.

Through Suitable surface identification module, PV-sim identifies also the available surfaces for deploying PV arrays on pitched rooftops excluding, for instance, dormers and chimneys.

To estimate the incident global radiation on pitched rooftops in real-sky conditions, both Direct Normal Incident radiation (DNI) and Diffuse Horizontal Incident radiation (DHI) are needed, as reported in [27]. Usually, weather stations in our city (e.g. [25]) are equipped with Global Horizontal solar radiation (GHI) sensors. To overcome this limitation, PVSim embeds the Solar radiation decomposition module. It integrates six solar decomposition models in literature [28][33] to calculate both DNI and DHI starting from GHI. Real-sky calculation module computes real-sky conditions and produces maps of incident global radiation on pitched rooftops.

Finally, the $P V$ energy estimation module computes the generation profiles of deployable PV systems with 15 minutes time interval for each area identified by previous modules. This module integrates weather information in order to model the effects of eviromental variables on PV cell efficency. Starting from the ambient temperature $T_{a}$, it computes the sol-air temperature $T_{\text {sol-air }}$ that is $T_{a}$ plus the loss factor $k$ (see Formula (1)) [34], [35]. Then, it estimates the cell temperature by using one of the following models: i) NOCT [36], if wind speed is not provided by the nearest weather station (see Formula (2) where $\tau \alpha=0.9$ as assumed by [37]) or ii) Mattei [38], if also information on wind speed are available (see Formula (3) where $\tau \alpha=0.81$ ). Finally, the instant power $P_{\text {out }}$ is computed by Formula 5 where characteristics of some commercial PV modules are given as default values (i.e. $\alpha_{p}, \eta_{m p, S T C}, T_{c, N O C T}, T_{c, S T C}, G_{t, N O C T}, \alpha_{\text {roof }}$ and $\left.h_{c}\right)$. The final output of the PV energy estimation module is a GeoJSON that provides for each building information on the size of deployable PV system and the related generation profiles for the requested time interval. These final results are retrieved every 15 minutes by the real-time simulator through the Communication Adapter. It exploits the REST web services provided by the $P V$ energy estimation module to feed the grid simulation strategy.

\section{Control strategy for battery management}

A distributed storage management strategy to control local energy balance in substations with high penetration of PV generation is modelled as a use case to test the implemented co-simulation architecture. The local controller reads the total amount of active power exchange in MV/LV transformers and 


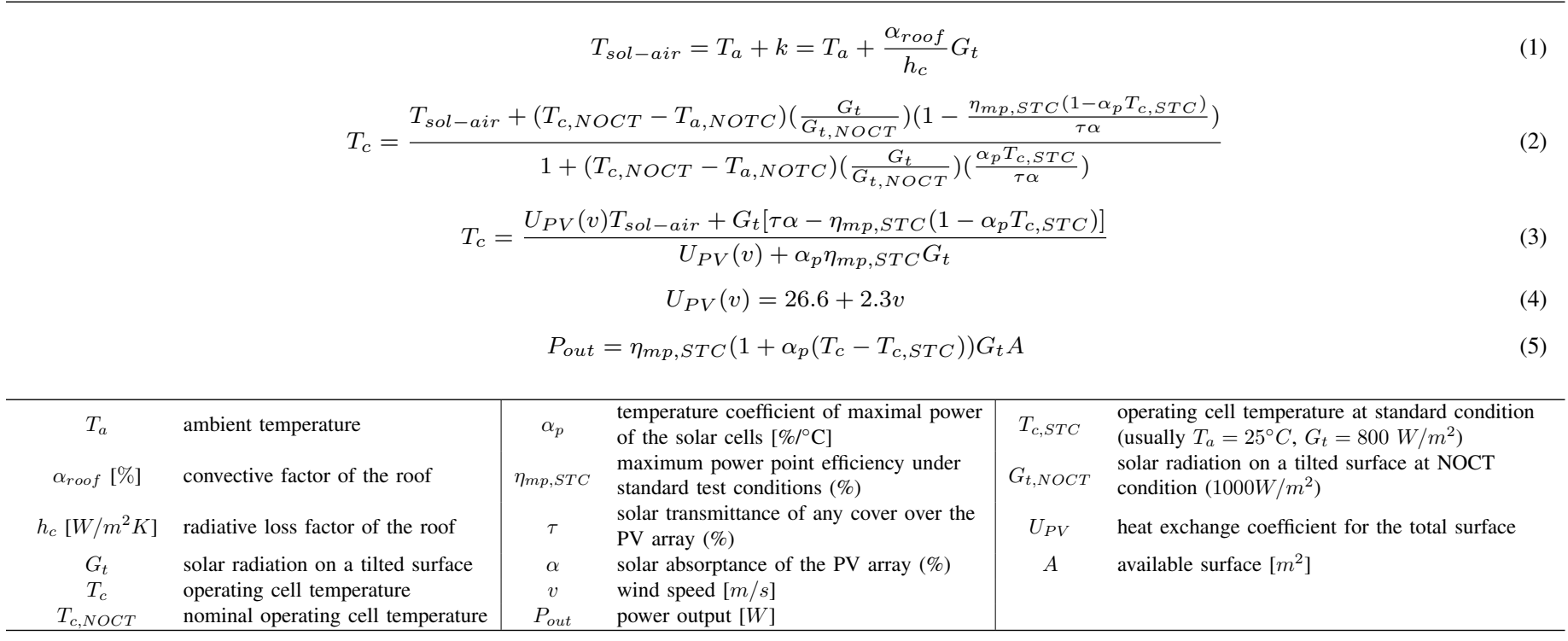

calculates the required amount of active power to be injected or absorbed by batteries connected on the LV side of the secondary substation. The transformer power measurement is periodically (every 15 minutes) sent to the battery controller module through MQTT protocol in our co-simulation infrastructure, and the battery power absorption or injection amount (i.e. charge or discharge respectively) is periodically pushed into the battery model inside the grid again through MQTT. In the grid simulator, batteries are modelled as dynamic loads with either positive (consumption mode) or negative (generation mode) power.

The modelled batteries are distributed stationary bulk storages connected to the LV side of the secondary substations, however the same platform can be used to simulate V2G by only changing battery models and battery control strategies.

The battery controller considers the batteries with the characteristics of lead-acid type. The control strategy is to compensate the power difference between the substation net consumption and zero as the reference value of an ideal micro-grid. Nominal power of the batteries, nominal capacity, efficiency and minimum-maximum state-of-charge (SOC) of the batteries are taken into account to calculate the right amount of charge/discharge power.

In order to create a soft transition between the battery on/off states, when the batteries reach the minimum and maximum SOC limits, the controller creates a ramp to proportionally decrease rate of charge or discharge. In this way the occurrence of a high step on the total net consumption of the substation is prevented.

\section{Case Study and Results}

The case study area involves an urban district where the DSM, with a resolution of $0.5 \mathrm{~m}^{2}$, and the MV grid topology are available. This district is located in Northern Italy and counts about 2200 residential buildings.

The MV grid in the district (Figure 6) consists of a primary substation with three $22 \mathrm{kV}$ busbars, each of which is fed by a transformer characterized by voltage ratio of $220 \mathrm{kV} / 22 \mathrm{kV}$. 43 substations are supplying loads (mainly residential buildings). These substations are equipped with a MV/LV transformer characterized by voltage ratio of $22 \mathrm{kV} / 400 \mathrm{~V}$ and a nominal power of $400 \mathrm{kVA}$ or $250 \mathrm{kVA}$. The total length of MV lines, mostly constituted by underground cables, is around $39 \mathrm{~km}$.

To evaluate the integration of PV systems and distributed storage in the district, a summer day scenario (in July) with a quite high PV production has been simulated. During summer days, in Italy, the energy consumption of residential users is lower than in winter days because residential households do not usually have air conditioning systems [39]. In addition, sunny days in summer produce more electricity from PV systems and this can be a critical situation for distribution grids. Figure 7 shows, as an example, the daily power profile for load and generation of one of the substations (204171). As previously described, the load profiles are generated based on some measurement data-sets we collected by real meters in some secondary substations. The generation profiles, instead, are produced by the photovoltaic system simulator. It exploits meteorological information retrieved by a weather station located in the urban district under analysis.

In this case study, we test the usefulness and functionality of the proposed distributed infrastructure for developing and testing distributed battery management when PV panels are installed on rooftops in the whole district area. The PV generation module does not consider grid constraints and introduces PV generation with the highest possible penetration, considering the available surface areas on the rooftops. In this case, choosing storage batteries with the right capacity, location, and charge/discharge strategy in not only beneficial but also a need.

In 9 substations, daily load and generation profiles are in such a way that the net consumption in some hours become negative. We added batteries on the LV side of the transformers in these 9 substations (positions are shown in Figure 6 with aggregated batteries) and we tested a control strategy to manage their charge/discharge profiles.

The added storage is constituted, at each substation, by 


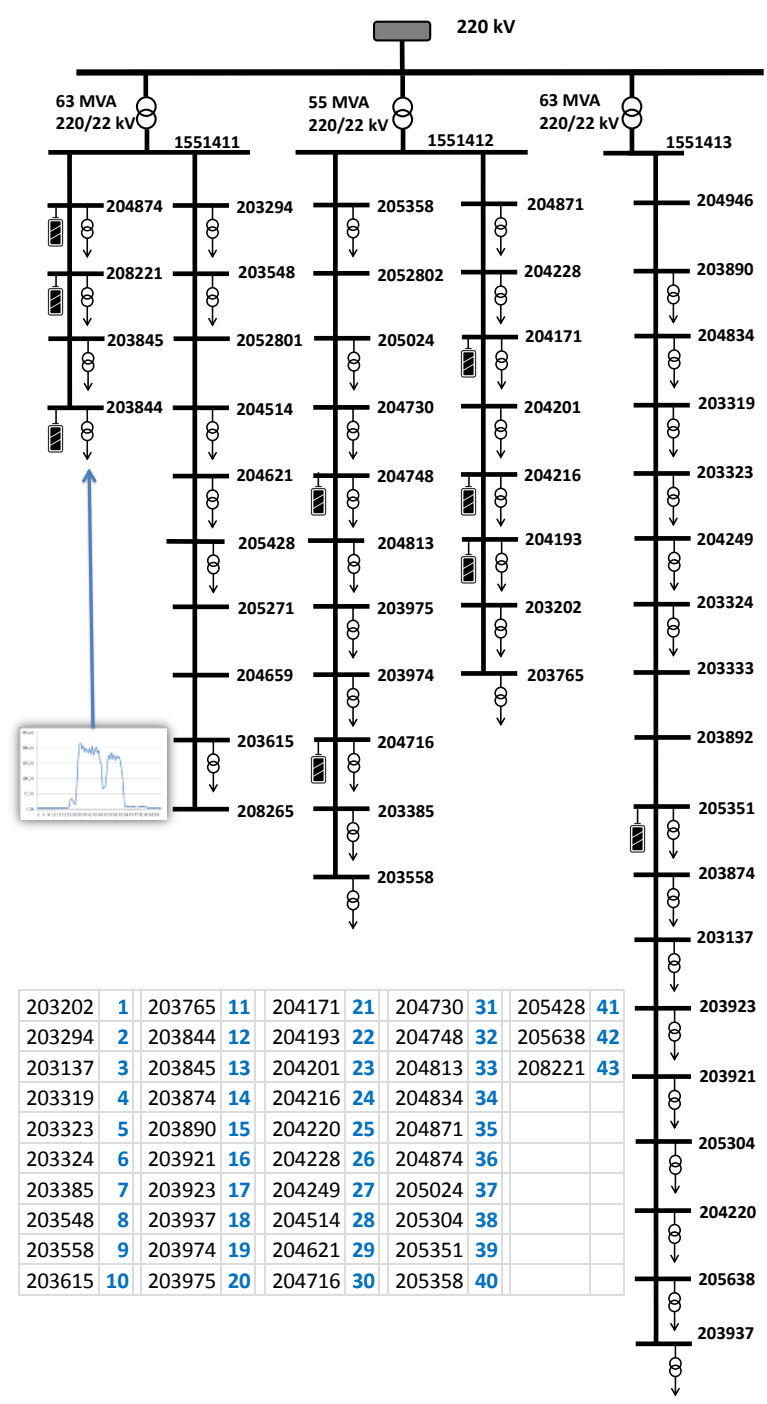

Fig. 6. Topology of a portion of MV distribution grid simulated in the case-study

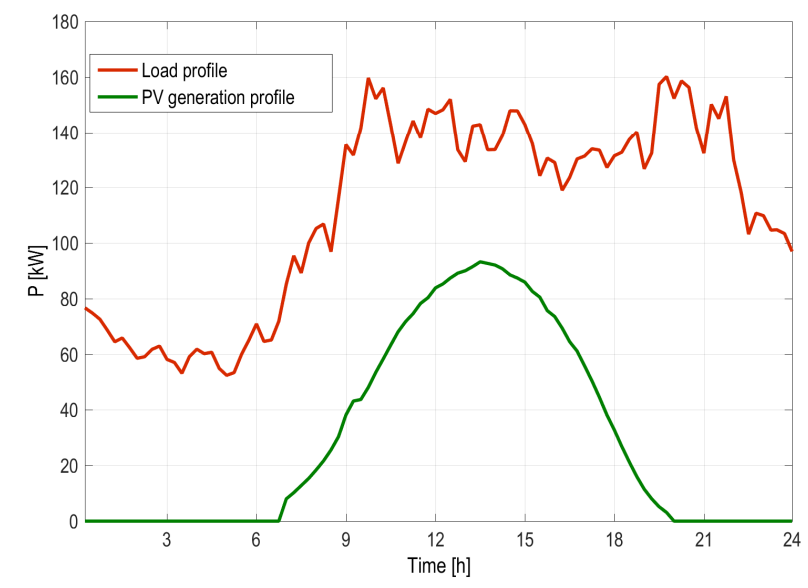

Fig. 7. Aggregated load and generation profiles in substation 204171 before battery installation

lead-acid batteries with a total capacity of 3000 Ah. The maximum power that each storage can exchange with the grid is $50 \mathrm{~kW}$, working between the limits of $10 \%$ and $100 \%$ SOC.

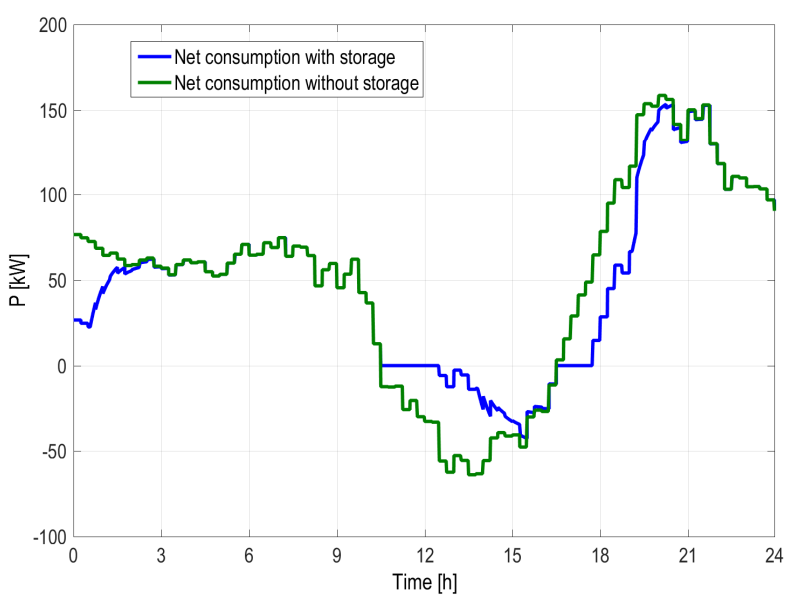

Fig. 8. Net consumption power with and without storage in substation 204171

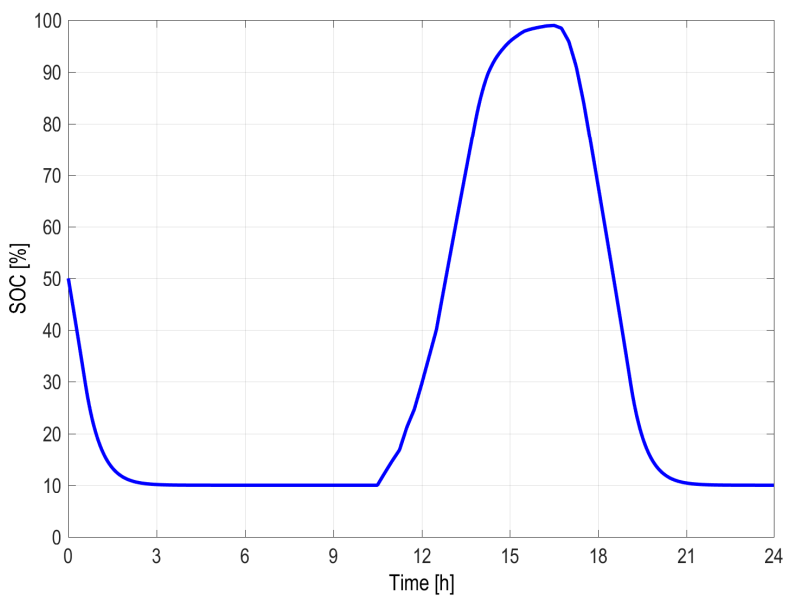

Fig. 9. State Of Charge profile of storage in substation 204171

Based on the control strategy explained in Section IV-D, the battery behaviour in terms of power exchange with the grid can be captured as a time-variant profile. For the same substation presented in Figure 7, in Figure 8 and Figure 9 we presented the net consumption with and without storage and the storage SOC respectively. In the simulation the storage initial SOC is $50 \%$. It initially discharges, as the net consumption in the substation is positive. When generation becomes bigger then load, the storage system charges trying to follow exactly the net consumption. At a certain point the residual power becomes negative as the maximum power of the storage is exceeded and because the SOC reaches $100 \%$. The smooth charging/discharging profile produced by the strategy under test can be observed in Figure 9. The areas between the two curves in Figure 8 represent the energy exchanged between storage and grid during the charge/discharge cycles: as batteries efficiency is kept into account in the model, discharge areas are smaller.

Similarly to what is presented in Figure 7, Figure 8 and Figure 9, in all the substations where storage is integrated, the net consumption curves are smoothed: during the night, when the substation net consumption is positive, batteries discharge until their SOC reaches the minimum level. The storages start 

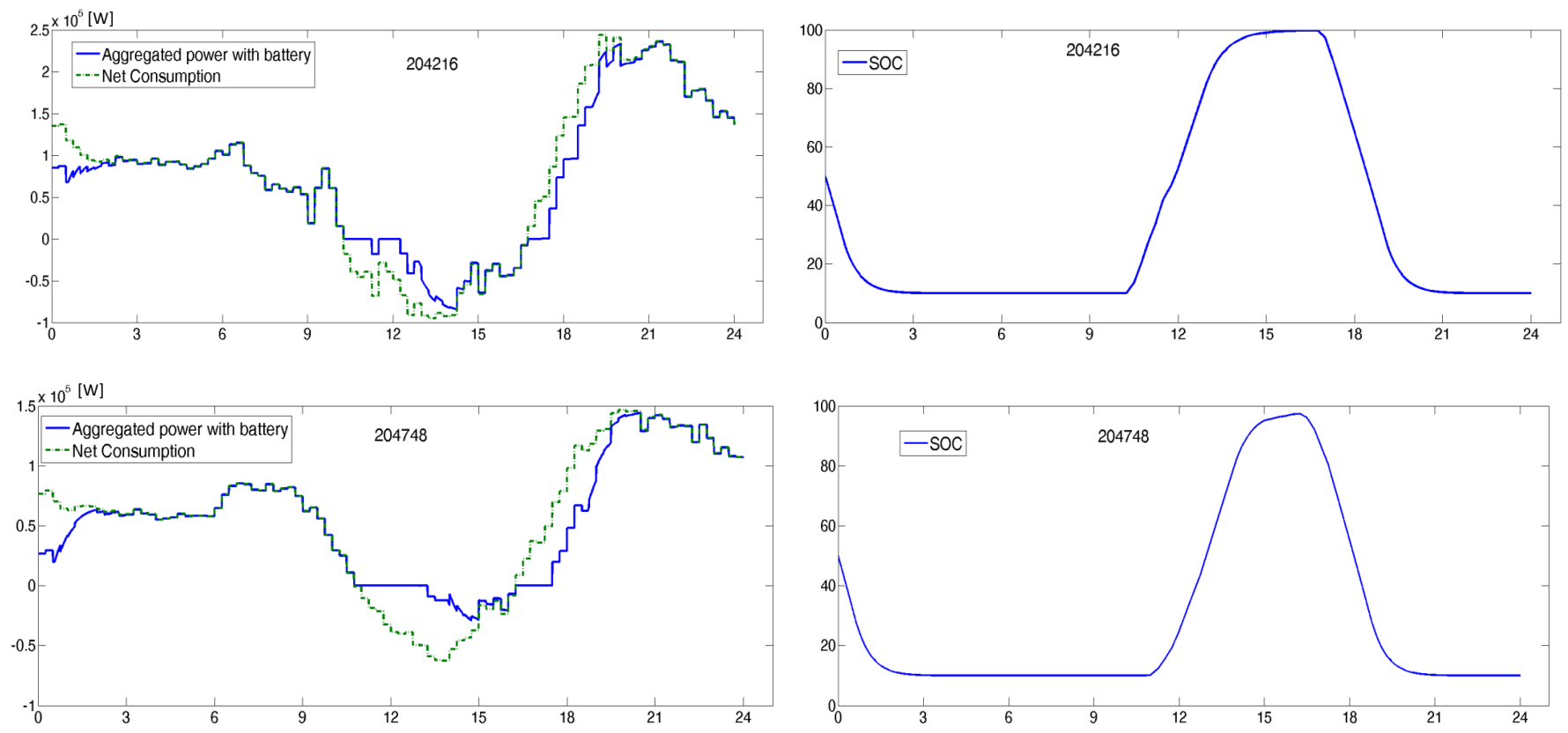

Fig. 10. Net consumption power with/without storage in [W] and SOC in [\%] in substations 204216 and 204748 during the day [h]

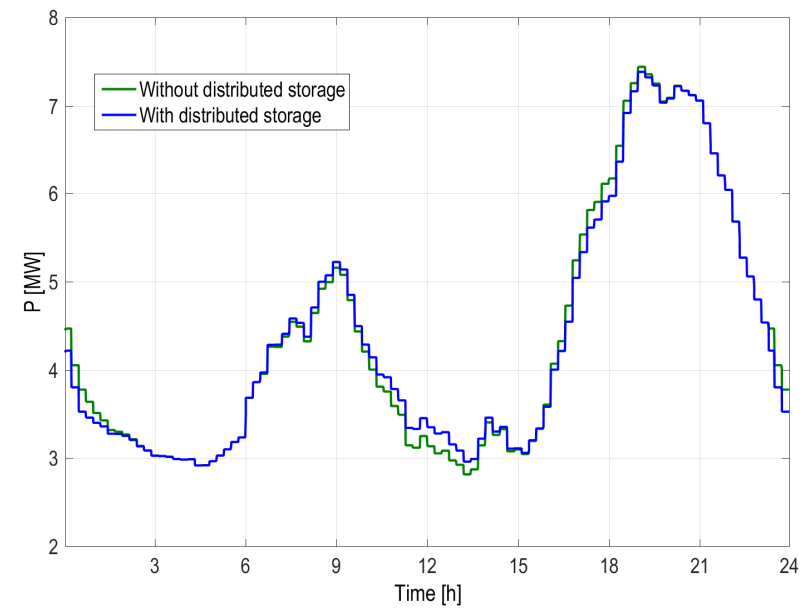

Fig. 11. Net demand profile of primary substation with and without storage

charging again when there is energy surplus in the substation or, in other words, the net consumption becomes negative. The results of two more substation are presented in Figure 10.

Thanks to the network model running in the real-time simulator, it is possible to analyse the impact of the storage control strategy not only on the single MV/LV substations, but also on the overall distribution grid. The profile of the net demand of the primary substation is shown in Figure 11.

\section{CONCLUSION}

In this paper, we presented a novel flexible and modular distributed infrastructure, based on real-time simulation, for multi-purpose Smart Grid studies. Hence, it is flexible in simulating different scenarios in power grids without affecting the whole infrastructure. It exploits a Communication Adapter that implements both publish/subscribe (MQTT) and request/response (REST) paradigms for communication and data exchange among the real-time simulator and different external modules. These modules can be software components, IoT devices, or other HW/SW directly connected to the realtime simulation for HIL or SIL. Both publish/subscribe and request/response are two communication models peculiar of IoT platforms, such as future Smart Metering Infrastructures. Thus, the proposed solution is also flexible i) to retrieve information in (near-) real-time from real IoT devices (i.e. smart meters) installed along the distribution network and ii) to feed strategies and models with real data during their running time.

An exemplification is presented and applied to the study of PV and storage integration in the distribution network of an urban district. The proposed example is simple but it is useful to demonstrate how the different distributed modules of the infrastructure can interact and exchange data, cooperating for obtaining the final results which is expected from an integrated co-simulation. Different load and generation scenarios can be easily fed into the whole simulation independently and also during running simulation. Storage control strategies can be tested as well without being locally integrated inside the grid model.

\section{REFERENCES}

[1] L. Dusonchet and E. Telaretti, "Economic analysis of different supporting policies for the production of electrical energy by solar photovoltaics in western european union countries," Energy Policy, vol. 38, no. 7, pp. 3297-3308, 2010.

[2] K. Kauhaniemi and L. Kumpulainen, "Impact of distributed generation on the protection of distribution networks," in Proc. of Developments in Power System Protection. Eighth IEEE International Conference on, vol. 1. IET, 2004, pp. 315-318.

[3] F. Bastiao, P. Cruz, and R. Fiteiro, "Impact of distributed generation on distribution networks," in Proc. of 5th International Conference on the European Electricity Market. IEEE, 2008, pp. 1-6. 
[4] R. C. Green, L. Wang, and M. Alam, "The impact of plug-in hybrid electric vehicles on distribution networks: A review and outlook," Renewable and Sustainable Energy Reviews, vol. 15, no. 1, pp. 544553, 2011.

[5] E. Bompard, B. Han, M. Masera, and E. Pons, "Smart grid as multilayer interacting system for complex decision makings," in Networks of Networks: The Last Frontier of Complexity. Springer, 2014, pp. 187-201.

[6] H. Georg, S. C. Müller, C. Rehtanz, and C. Wietfeld, "Analyzing cyberphysical energy systems: The inspire cosimulation of power and ict systems using hla," IEEE Trans. on Industrial Informatics, vol. 10, no. 4, pp. 2364-2373, 2014.

[7] C.-h. Yang, G. Zhabelova, C.-W. Yang, and V. Vyatkin, "Cosimulation environment for event-driven distributed controls of smart grid," IEEE Trans. on Industrial Informatics, vol. 9, no. 3, pp. 1423-1435, 2013.

[8] M. Manbachi, A. Sadu, H. Farhangi, A. Monti, A. Palizban, F. Ponci, and S. Arzanpour, "Real-time co-simulation platform for smart grid volt-var optimization using iec 61850," IEEE Trans. on Industrial Informatics, vol. 12, no. 4, pp. 1392-1402, Aug 2016.

[9] L. Bottaccioli, A. Estebsari, E. Patti, E. Pons, and A. Acquaviva, "A novel integrated real-time simulation platform for assessing photovoltaic penetration impacts in smart grids," Energy Procedia, vol. 111, pp. 780 789, 2017.

[10] L. Bottaccioli, E. Macii, E. Patti, A. Estebsari, E. Pons, and A. Acquaviva, "Pvingrid: A distributed infrastructure for evaluating the integration of photovoltaic systems in smart grid," in Proc. of 8th Advanced Doctoral Conference on Computing, Electrical and Industrial Systems. Springer, 2017.

[11] S. Schütte, S. Scherfke, and M. Tröschel, "Mosaik: A framework for modular simulation of active components in smart grids," in Proc. of SGMS11. IEEE, 2011, pp. 55-60.

[12] S. Rohjans, S. Lehnhoff, S. Schtte, S. Scherfke, and S. Hussain, "mosaik - a modular platform for the evaluation of agent-based smart grid control," in Proc. of PES ISGT, Oct 2013, pp. 1-5.

[13] T. Hess, J. Dickert, and P. Schegner, "Multivariate power flow analyses for smart grid applications utilizing mosaik," in Proc. of PES ISGT, Oct 2016, pp. 1-6.

[14] K. Anderson, J. Du, A. Narayan, and A. El Gamal, "Gridspice: A distributed simulation platform for the smart grid," IEEE Trans. on Industrial Informatics, vol. 10, no. 4, pp. 2354-2363, 2014.

[15] A. Awad, P. Bazan, and R. German, "Sgsim: Co-simulation framework for ict-enabled power distribution grids," in Proc. of $M M B$ \& $D F$. Springer, 2016, pp. 5-8.

[16] V. Venkataramanan, A. Srivastava, and A. Hahn, "Real-time cosimulation testbed for microgrid cyber-physical analysis," in Proc. of MSCPES, April 2016, pp. 1-6.

[17] M. Brenna, E. De Berardinis, L. D. Carpini, F. Foiadelli, P. Paulon, P. Petroni, G. Sapienza, G. Scrosati, and D. Zaninelli, "Automatic distributed voltage control algorithm in smart grids applications," IEEE Trans. on Smart Grid, vol. 4, no. 2, pp. 877-885, 2013.

[18] A. Estebsari, E. Pons, E. Patti, M. Mengistu, E. Bompard, A. Bahmanyar, and S. Jamali, "An iot realization in an interdepartmental real time simulation lab for distribution system control and management studies," 2016.

[19] P. T. Eugster, P. A. Felber, R. Guerraoui, and A.-M. Kermarrec, "The many faces of publish/subscribe," ACM CSUR, June 2003.

[20] E. Patti, A. L. A. Syrri, M. Jahn, P. Mancarella, A. Acquaviva, and E. Macii, "Distributed software infrastructure for general purpose services in smart grid," IEEE Trans. on Smart Grid, vol. 7, no. 2, pp. 1156-1163, March 2016.

[21] E. Patti, E. Pons, D. Martellacci, F. B. Castagnetti, A. Acquaviva, and E. Macii, "multiflex: Flexible multi-utility, multi-service smart metering architecture for energy vectors with active prosumers," in Proc. of SMARTGREENS, May 2015, pp. 1-6.

[22] MQTT, Accessed April 2017. [Online]. Available: http://mqtt.org

[23] R. T. Fielding, Architectural styles and the design of network-based software architectures, Open Source Geospatial Foundation, 2000.

[24] E. Bompard, A. Monti, A. Tenconi, A. Estebsari, T. Huang, E. Pons, M. Stevic, S. Vaschetto, and S. Vogel, "A multi-site real-time cosimulation platform for the testing of control strategies of distributed storage and v2g in distribution networks," in Proc. of EPE'16 ECCE. IEEE, 2016, submitted for publication.

[25] Weather Underground. [Online]. Available: http://www.wunderground.com/

[26] M. Fowler, "Microservices," 2014. [Online]. Available: http://martinfowler.com/articles/microservices.html
[27] M. Š́ri and J. Hofierka, "A new gis-based solar radiation model and its application to photovoltaic assessments," Trans. in GIS, vol. 8, no. 2, pp. 175-190, 2004.

[28] D. Erbs, S. Klein, and J. Duffie, "Estimation of the diffuse radiation fraction for hourly, daily and monthly-average global radiation," Solar Energy, vol. 28, no. 4, pp. 293-302, 1982.

[29] N. Engerer, "Minute resolution estimates of the diffuse fraction of global irradiance for southeastern australia," Solar Energy, vol. 116, pp. 215 237, 2015.

[30] J. Ruiz-Arias, H. Alsamamra, J. Tovar-Pescador, and D. Pozo-Vzquez, "Proposal of a regressive model for the hourly diffuse solar radiation under all sky conditions," Energy Conversion and Management, vol. 51, no. 5, pp. $881-893,2010$.

[31] S. Karatasou, M. Santamouris, and V. Geros, "Analysis of experimental data on diffuse solar radiation in athens, greece, for building applications," International journal of sustainable energy, vol. 23, no. 1-2, pp. 1-11, 2003.

[32] D. T. Reindl, W. A. Beckman, and J. A. Duffie, "Diffuse fraction correlations," Solar energy, vol. 45, no. 1, pp. 1-7, 1990.

[33] A. Skartveit and J. A. Olseth, "A model for the diffuse fraction of hourly global radiation," Solar Energy, vol. 38, no. 4, pp. 271-274, 1987.

[34] J. A. Jakubiec and C. F. Reinhart, "A method for predicting city-wide electricity gains from photovoltaic panels based on lidar and gis data combined with hourly daysim simulations," Solar Energy, vol. 93, pp. 127-143, 2013.

[35] L. R. Camargo, R. Zink, W. Dorner, and G. Stoeglehner, "Spatiotemporal modeling of roof-top photovoltaic panels for improved technical potential assessment and electricity peak load offsetting at the municipal scale," Comput. Environ. Urban Syst., vol. 52, pp. 58-69, 2015.

[36] F. Brihmat and S. Mekhtoub, "Pv cell temperature/pv power output relationships homer methodology calculation," in Proc. of CIER' 13, vol. 1, no. 02. International Publisher \&C. O, 2014.

[37] J. A. Duffie and W. A. Beckman, Solar Engineering of Thermal Processes. Wiley, 2013.

[38] M. Mattei, G. Notton, C. Cristofari, M. Muselli, and P. Poggi, "Calculation of the polycrystalline pv module temperature using a simple method of energy balance," Renewable energy, vol. 31, no. 4, pp. 553-567, 2006.

[39] S. Maggiore, "Analisi di impatto dell'introduzione della tariffa bioraria obbligatoria," Prot: 13000580 RSE SpA, 2012. [Online]. Available: http://doc.rse-web.it/doc/doc-sfoglia/13000580315241/13000580-315241.html 